\title{
Transmissible gastroenteritis virus targets Paneth cells to inhibit the self-renewal and differentiation of Lgr5 intestinal stem cells via Notch signaling
}

Aimin $W u^{1,2}$, Bing $Y u^{1,2}$, Keying Zhang ${ }^{1,2}$, Zhiwen $X u^{3}$, De $W u^{1,2}$, Jun He $\mathbb{D}^{1,2}$, Junqiu Luo ${ }^{1,2}, Y$ Yuheng Luo $0^{1,2}$, Jie $Y u^{1,2}$, Ping Zheng ${ }^{1,2}$, Lianqiang Che ${ }^{1,2}$, Xiangbing Mao ${ }^{1,2}$, Zhiqing Huang $\mathbb{1}^{1,2}$, Lan Wang ${ }^{1,2}$, Jun Zhao ${ }^{3}$ and Daiwen Chen ${ }^{1,2}$

\begin{abstract}
Infection with transmissible gastroenteritis virus (TGEV) has been associated with villous atrophy within $48 \mathrm{~h}$, which seriously disrupts intestinal homeostasis. However, the underlying mechanisms remain elusive. In this study, we found that TGEV infection severely disrupted intestinal homeostasis via inhibition of self-renewal and differentiation in Lgr5 intestinal stem cells (ISCS). Profoundly, TGEV-encoded NSP10/NSP16 protein complex-mediated the inactivation of Notch signaling provided a mechanistic explanation for this phenomenon. Initial invasions by TGEV-targeted Paneth cells through aminopeptidase N (APN) receptor, then inducing mitochondrial damage and ROS generation in them, ultimately causing Paneth cell decrease and loss of Notch factors (DII4 and Hes5), which are essential for Lgr5 ISCs selfrenewal and differentiation. Interestingly, loss of Notch signaling induced goblet cells differentiation at the cost of absorptive enterocytes and promoted mucins secretion, which accelerated TGEV replication. Therefore, the more differentiation of goblet cells, the greater TGEV infection in jejunum. These results provide a detailed mechanistic pathway by which villous atrophy sharply occurs in TGEV-infected jejunum within $48 \mathrm{~h}$. Thus, the pathogenesis of TGEV can be described as a "bottom up scenario", which is contrary to the traditional "top down" hypothesis. Together, our findings provide a potential link between diarrheal virus infection and crypt cells response that regulates Paneth cells function and Lgr5 ISCs fate and could be exploited for therapeutic application.
\end{abstract}

\section{Introduction}

The mammalian intestinal epithelium exhibits rapid self-renewal, with complete turnover of epithelial cell lining every $4-5$ days $^{1,2}$. In addition to its role in nutrient digestion and absorption, the intestinal epithelium also forms as a critical barrier against luminal pathogens to maintain intestinal homeostasis ${ }^{3}$. However, this barrier function is known to be susceptible to irradiation, chemical injury or infection with pathogens ${ }^{4}$. One of the central mechanisms to maintain intestinal barrier

\footnotetext{
Correspondence: Daiwen Chen (dwchen@sicau.edu.cn)

${ }^{1}$ Institute of Animal Nutrition, Sichuan Agricultural University, Chengdu, China

${ }^{2}$ Key Laboratory for Animal Disease-resistance Nutrition of China Ministry of Education, Sichuan Agricultural University, Chengdu, China

Full list of author information is available at the end of the article.

These authors contributed equally: Aimin Wu, Bing Yu

Edited by A. Stephanou
}

function is through activating crypt cells (Paneth cells and intestinal stem cells, ISCs) ${ }^{5}$. Whereas the mechanism by which irradiation or chemical toxins activate crypt cells response has been extensively studied ${ }^{4,6}$, far less is known about the response of crypt cells to diarrheal virus infection, such as TGEV.

TGEV, together with the human coronaviruses (HCoVNL63 and HCoV-229e), belongs to the genus Alphacoronavirus within the subfamily Coronaviridae ${ }^{7}$, which are single-stranded, positive-sense RNA viruses relevant in animal and human health ${ }^{8}$. Generally, TGEV causes transmissible gastroenteritis with high morbidity in pigs of all ages and as high as $100 \%$ mortality in newborn piglets, especially those within 2 weeks of birth ${ }^{9}$. Most notably, TGEV not only causes devastating impact on the global pig industry, but also is a potential threat to human

\section{(c) The Author(s) 2020}

(c) (i) Open Access This article is licensed under a Creative Commons Attribution 4.0 International License, which permits use, sharing, adaptation, distribution and reproduction cc) in any medium or format, as long as you give appropriate credit to the original author(s) and the source, provide a link to the Creative Commons license, and indicate if changes were made. The images or other third party material in this article are included in the article's Creative Commons license, unless indicated otherwise in a credit line to the material. If material is not included in the article's Creative Commons license and your intended use is not permitted by statutory regulation or exceeds the permitted use, you will need to obtain permission directly from the copyright holder. To view a copy of this license, visit http://creativecommons.org/licenses/by/4.0/. 
health, as its infection suppresses protein translation in diverse human cells ${ }^{10,11}$. The economic and health implications of TGEV have aroused significant concern worldwide? .

The typical symptomatic pathway of TGEV infection is villous atrophy within $48 \mathrm{~h}$, followed by crypt hyperplasia, concomitant with lethal watery diarrhea, and ultimately severe dehydration in piglets until death ${ }^{12}$. TGEV infection causes intestinal barrier dysfunction and disrupts intestinal homeostasis ${ }^{9,13}$, which could affect intestinal epithelium renewal. The intestinal homeostasis and intestinal epithelium renewal are normally sustained by fast-cycling stem cells located around the base of the crypt $^{14}$. These internal cycling stem cells are distinguished by Lgr 5 expression, which is suggested to mediate cell proliferation in a number of tissues ${ }^{15}$. Small populations of Lgr5 ISCs regularly divide to produce highly proliferative progenitors known as transit-amplifying (TA) cells $^{14}$ and terminally differentiate into the absorptive (enterocytes) or secretory (Paneth cells, goblet cells and enteroendocrine cells) cell lineages while gradually migrating upwards toward the top of the villi ${ }^{16}$. One exception to this migratory path is Paneth cells, which follow a downward migratory path to the crypt bottom and intermingle with Lgr5 ISCs ${ }^{17}$. The close interaction of Lgr5 ISCs and Paneth cells is essential for maintaining Lgr5 ISCs fate ${ }^{18}$, since Paneth cells provide crucial niche factors (such as Wnt3a, BMP, and Notch factors) for Lgr5 ISCs self-renewal and differentiation ${ }^{19}$. Of particular significance, Notch factors regulate TA differentiation status and intestinal developmental pattern ${ }^{20}$. Paneth cells express Notch ligands, which bind the Notch receptor on Lgr5 ISCs to activate expression of downstream genes, such as Hes1 and Hes $5^{21,22}$. These Notch target genes are essential for ISCs homeostasis, as inhibition of the Notch signaling results in complete conversion of epithelial cells into goblet cells both in vitro and in vivo ${ }^{23,24}$. In contrast, activation of the Notch signaling promotes proliferation to the absorptive cell lineage with a concomitant loss in secretory cell lineage differentiation ${ }^{25}$. Among Notch ligands, DII1 and DII4 are essential, and inhibition of both results in the loss of stem and progenitor cells ${ }^{22}$.

In this study, we explore how TGEV infection targets Paneth cells and through them disrupt Lgr5 ISCs and their ability to regenerate and differentiate, as well as adjacent effects on goblet cells and their role in maintaining the protective lining of the intestinal epithelium. We challenged the assumption that TGEV disruption of the jejunum occurs via a "top down" pathway with direct effects on the crypt cells, and instead consider how TGEV may function as a "bottom up" scenario with infection of Paneth cells initiating a cascade of events that ultimately inhibit intestinal epithelial homeostasis. These findings revealed a previously unrecognized link between diarrheal virus and crypt cells response that modulates ISCs homeostasis and could be exploited for therapeutic application.

\section{Results \\ TGEV infection disrupts intestinal homeostasis}

As TGEV major targets organ is small intestine. Therefore, we collected the duodenum, jejunum and ileum tissues for intestinal architecture analysis. Histological analysis of these samples revealed that TGEV significantly disrupted intestinal morphology in small intestine, especially in jejunum (Fig. 1a). The most striking features were villous atrophy. Villous height decreased sharply, accompanying with enterocyte shedding, followed by crypt hyperplasia (Fig. 1a, b). Subsequently, the representative tight junction proteins Zo-1, Occludin, and Claudin-1 were determined by using western blot (WB). The results indicated that TGEV solely down-regulated Zo-1 protein level in infected jejunum (Fig. 1c, d). Moreover, we found that TGEV infection robustly halted cell proliferation both in crypt and villous (Fig. 1e, f), as assessed by Ki67 staining. Unlike cell proliferation, TGEV induced cell apoptosis solely in crypt cells, as detected by TUNEL staining (Fig. 1g, h). These results also were confirmed in IPEC-J2 cells (a jejunal epithelial cell line). TGEV infection not only inhibited cell proliferation, but also induced cell apoptosis in $36 \mathrm{~h}$ post infected cells (Supplementary Fig. S1). Together, these data strongly suggest that TGEV infection inhibits epithelial selfrenewal and disrupts intestinal homeostasis.

\section{TGEV infection inhibits the self-renewal of Lgr5 ISCs and the differentiation of absorptive lineage}

To test the hypothesis that TGEV infection disruption intestinal homeostasis through inhibiting the self-renewal and differentiation of Lgr5 ISCs, WB, FACS, FISH and IF staining were used to determine the self-renewal of Lgr5 ISCs in TGEV-infected jejunum. We observed a significant decrease in Lgr5 ISCs number within crypt tissues for TGEV-infected jejunum (Fig. 2a, b). Similarly, Olfm4, another marker of ISCs, was repressed (Fig. 2a-d). In parallel, similar results were found in TGEV-infected IPEC-J2 cells (Supplementary Fig. S2a, b). We then examined the differentiation pattern of Lgr5 ISCs in TGEV-infected jejunum. The number of enterocytes was distinctly decreased in TGEV-infected jejunum, as revealed by SI protein expression (Fig. 2c, d). Similar results were determined in Paneth cells (Fig. 2e, f). Moreover, TGEV infection not only decreased the number of Paneth cells in crypts, but also caused enteroendocrine cells loss in crypts and villi (Fig. 2c, d, g, and h). In contrast, Muc2 protein level and PAS staining showed that TGEV infection strongly increased the number of goblet cells both in crypts and villi (Fig. 2c, d, i, and j). Subsequently, we found that TGEV infection not 


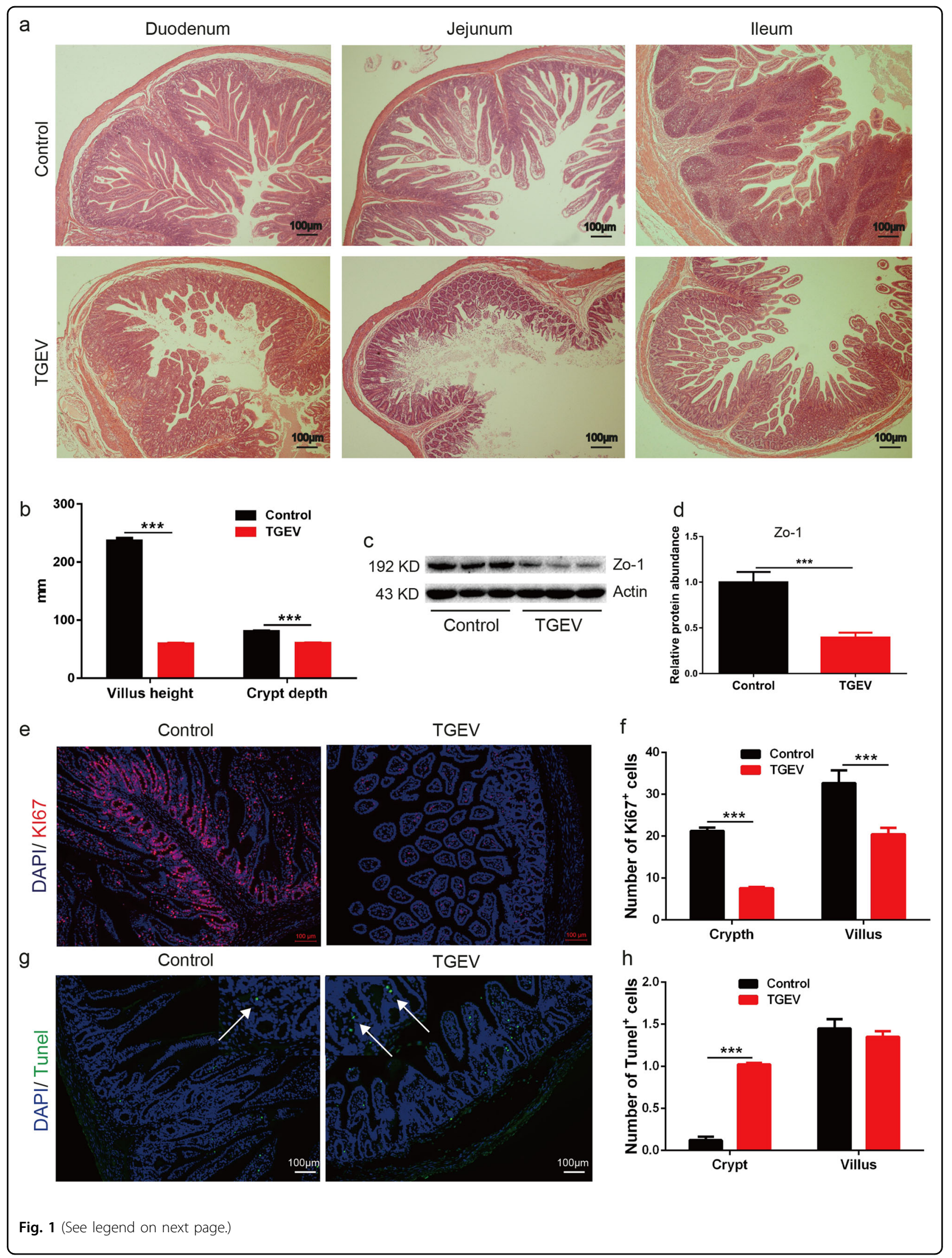


(see figure on previous page)

Fig. 1 TGEV infection destroys the integrity of intestinal architecture and disrupts intestinal homeostasis. a Representative H\&E stained crosssection showing villous atrophy and enterocytes shedding in TGEV-infected intestine. Scale bar, $100 \mu \mathrm{m}$. b Quantification of villous height $(n=150)$ and crypt depth $(n=150)$ in TGEV-infected jejunum $(n=3)$. c Western blot for junction protein Zo-1, Occludin, and Claudin-1 of jejunum from control and TGEV-infected piglets. Actin serves as a control. $\mathbf{d}$ Quantitation of bands to demonstrate the protein level of Zo-1. e, $\mathbf{f}$ Jejunal cross-section stained with KI67 (Scale bars, $100 \mu \mathrm{m})$ and quantification of the proliferation cells per crypt $(n=150)$ and villus $(n=150)$. g Crosssection of jejunum from control and TGEV-infected piglets stained with Tunel (the white arrows show cell apoptosis in crypt; Scale bars, $100 \mu \mathrm{m}$ ). h Quantification of necrotic cells per crypt $(n=150)$ and villus $(n=150)$.

only halts cell proliferation and but also induces cell apoptosis in Lgr5 ISCs (Supplementary Fig. S2c, d). Together, TGEV infection induces goblet cells differentiation at the cost of absorptive enterocytes in TGEVinfected jejunum and severely alters Lgr5 ISCs fate.

\section{TGEV infection causes Paneth cells loss and disrupts the Notch signaling for Lgr5 ISCs self-renewal and differentiation}

To uncover the potential mechanisms for Lgr5 ISCs loss and the impact of TGEV infection on the niche signals for Lgr5 ISCs fate decision, we took advantage of TGEVinfected IPEC-J2 cells model. FACS for CD24 demonstrated that TGEV infection resulted in CD24 cell loss in TGEV-infected IPEC-J2 cells (Supplementary Fig. S3a), and IF staining results also revealed that TGEV infection decreased the expression of CD24 in TGEV-infected jejunum (Supplementary Fig. S3b, c). Subsequently, we found TGEV infection caused Paneth cell $\left(\mathrm{CD} 24^{+} \mathrm{SSC}^{\text {high }}\right.$ cells) and enteroendocrine cell (CD24 ${ }^{+} \mathrm{SSC}^{\text {low }}$ cells) loss (Supplementary Fig S3d; Fig. 3a, b), as was observed in TGEV-infected jejunum (Fig. 2e-h). We next examined apoptotic cell death and cell proliferation to determine potential mechanisms for Paneth cells loss with TGEV infection. Either early cell apoptosis or late cell apoptosis was robustly induced by TGEV in $\mathrm{CD} 24^{+} \mathrm{SSC}^{\text {high }}$ cells (Supplementary Fig. S4a, b). Meanwhile, similar results were detected in $\mathrm{CD}_{24}{ }^{+} \mathrm{SSC}^{\text {low }}$ cells (Supplementary Fig. S4c, d), but no changes in cell apoptosis were found in CD24- cells (Supplementary Fig. S4e, f). Cell apoptosis mainly occurred in $\mathrm{CD}_{2} 4^{+}$cells. Moreover, combining with FACS analysis of Paneth cells proliferation with Ki67 staining, TGEV infection inhibited the proliferation of Paneth cell $\left(\mathrm{CD} 24^{+} \mathrm{SSC}^{\text {high }}\right.$ cells) and enteroendocrine cell (CD24 ${ }^{+}$SSC $^{\text {low }}$ cells) in TGEV-infected IPEC-J2 cells (Supplementary Fig. S4g, h). Subsequently, we detected the mitochondrial function and ROS generation in TGEV-infected IPEC-J2 cells, which largely contribute to cell apoptosis and barrier functions of intestinal epithelial cells. As expected, TGEV infection significantly induced mitochondria damage and ROS production not only in Paneth cells $\left(\mathrm{CD} 24^{+} \mathrm{SSC}^{\text {high }}\right.$ cells) but also in enteroendocrine (CD24 ${ }^{+} \mathrm{SSC}^{\text {low }}$ cells) (Fig. $\left.4 \mathrm{a}, \mathrm{b}\right)$.
Since Paneth cells provide various niche factors to support ISCs self-renewal and differentiation. Paneth cells loss seriously affects the number and function of ISCs through reducing niche factor generation. To reveal the effect of TGEV on niche factor production, we first detected the mRNA expression of the representative niche factor markers in TGEV-infected jejunum (Fig. 3c) and IPEC-J2 cells (Fig. 3d). These results showed that TGEV infection significantly inhibited Notch ligand Dii4 and Notch effector Hes 5 mRNA expression. For Wnt (Wnt11) and BMP $(T g f-\beta)$ signaling, no significant changes in mRNA level were observed in TGEV-infected jejunum or IPEC-J2 cells (Fig. 3c, d). Then DII4 and Hes5 protein expression was quantified in TGEV-infected jejunum and IPEC-J2 cells by using WB (Fig. 3e-g). Infection by TGEV disrupted the Notch signaling for Lgr5 ISCs self-renewal and differentiation via down-regulating DII4 and Hes5 protein expression both in in vivo (Fig. 3e, f) and in vitro (Fig. 3g). In addition, TGEV infection decreased SI, CgA, CD24 protein expression (Fig. 3g). Alternatively, goblet cells (Muc2) were up-regulated in TGEV-infected IPECJ2 cells (Fig. 3g), with similar effect on goblet cells was detected in TGEV-infected jejunum (Fig. 2c, i, j). Subsequently, we inhibited Notch signaling in IPEC-J2 cells by using $N$-S-phenyl-glycine-t-butyl ester (DAPT), a $\gamma$ secretase inhibitor, which turns proliferative cells in intestinal crypts and adenomas into goblet cells. As expected, DAPT rapidly induced goblet cells differentiation at the cost of absorptive cells in IPEC-J2 cells (data not shown). Surprisingly, Notch signaling inhibition strongly promoted TGEV infection and replication in IPEC-J2 cells (Fig. 3h). Therefore, these results demonstrate that TGEV infection alters Lgr5 ISCs fate via inducing Paneth cells loss and disrupting Notch signaling factors. Moreover, goblet cells differentiation enhances TGEV infection and replication.

\section{Paneth cells $\left(\mathrm{CD} 24^{+} \mathrm{SSC}^{\mathrm{High}}\right)$ are the initial invasion cells of TGEV}

As noted earlier, TGEV infection distinctly affects the number and function of Paneth cells. To uncover the specific mechanism, we detected the infection proportion and replication of TGEV in IPEC-J2 cells. Of interest, 


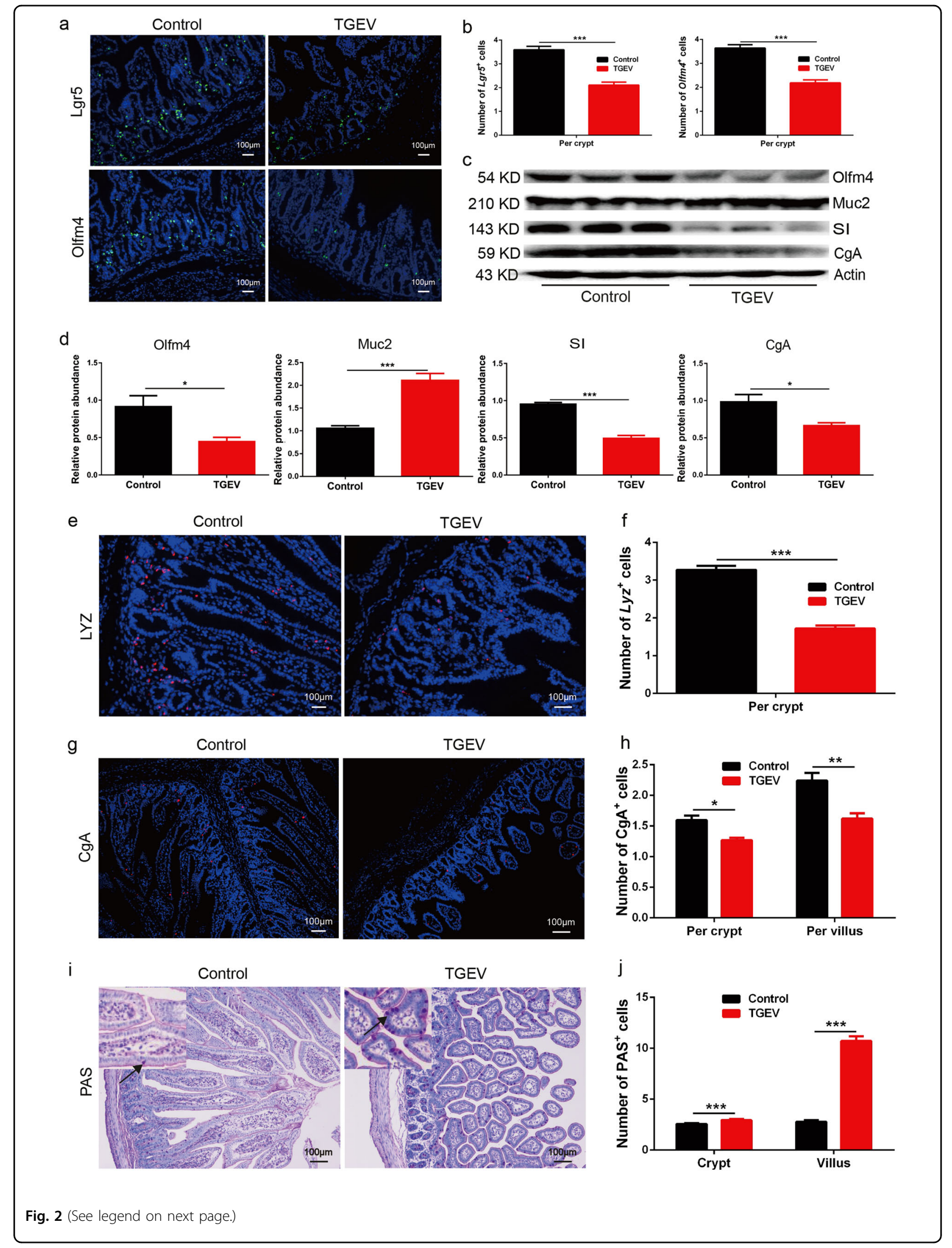


(see figure on previous page)

Fig. 2 TGEV infection inhibits the self-renewal of Lgr5 ISCs and the differentiation of absorptive lineage. a Fluorescence in situ hybridization with a probe for Lgr5 and Olfm4 performed on the jejunum, revealing TGEV infection decreases the mRNA expression of Lgr5 and Olfm4.

b Quantification of Lgr5 and Olfm4 stem cells number per crypt $(n=150)$. c Western blot for junction protein Olfm4, Muc2, SI, and CgA of jejunum from control and TGEV-infected piglets. Actin serves as a control. d Quantitation of bands to demonstrate the protein level of Olfm4, Muc2, SI and $\mathrm{CgA}$. e, $\mathbf{f}$ Fluorescence in situ hybridization with a probe for $L y z$ (Paneth cells) performed on the jejunum, and quantification of Paneth cells per crypt $(n=150)$. $\mathbf{g}$, h Jejunal cross-section stained with CgA shows enteroendocrine, and quantification of enteroendocrine per crypt $(n=150)$ and villus $(n=150)$. i, $\mathbf{j}$ Representative jejunal PAS stained cross-section, showing goblet cells (black arrows) and quantification of goblet cells per crypt $(n=150)$ and villus $(n=150)$, (Scale bars, $100 \mu \mathrm{m})$.

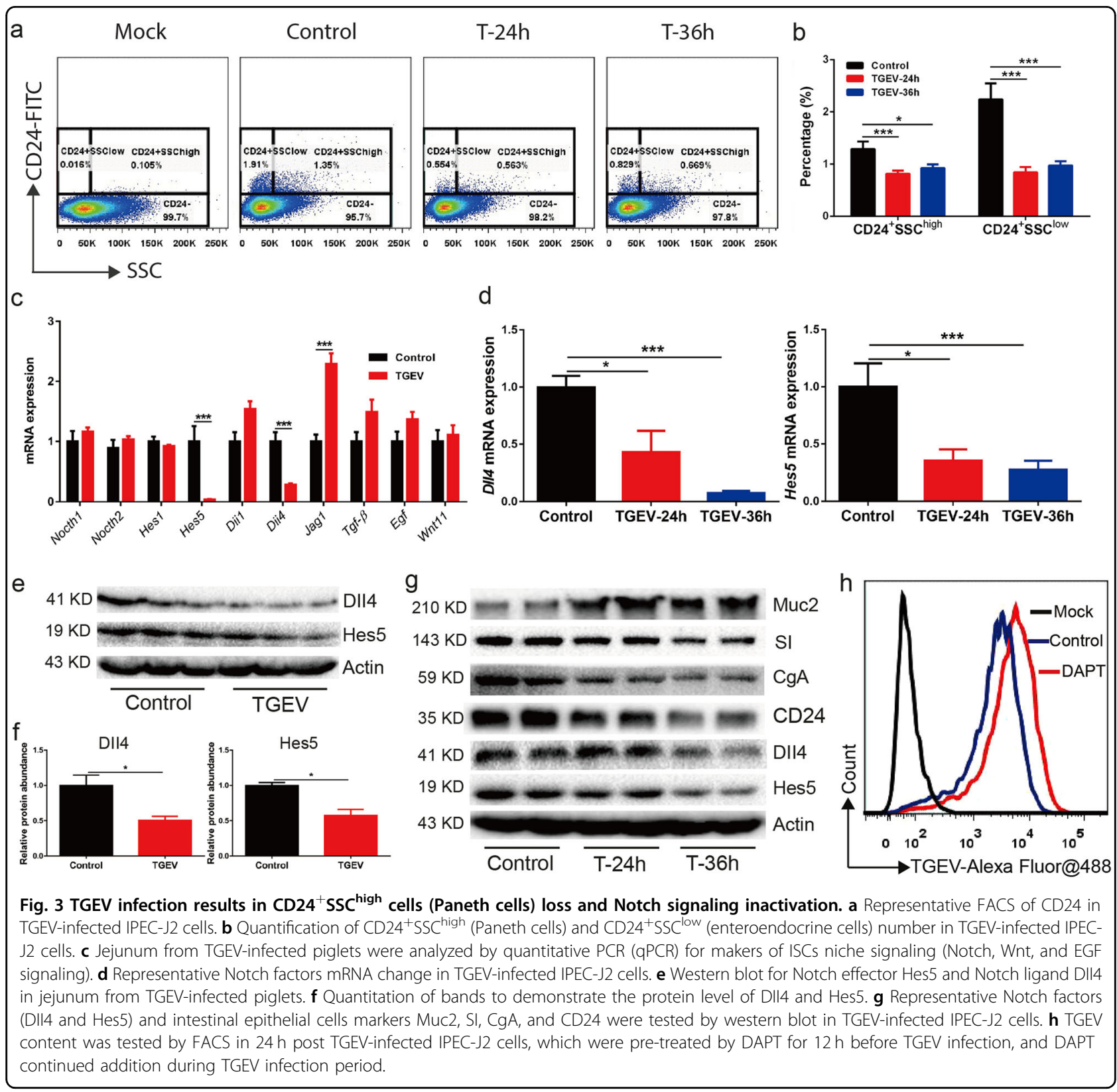




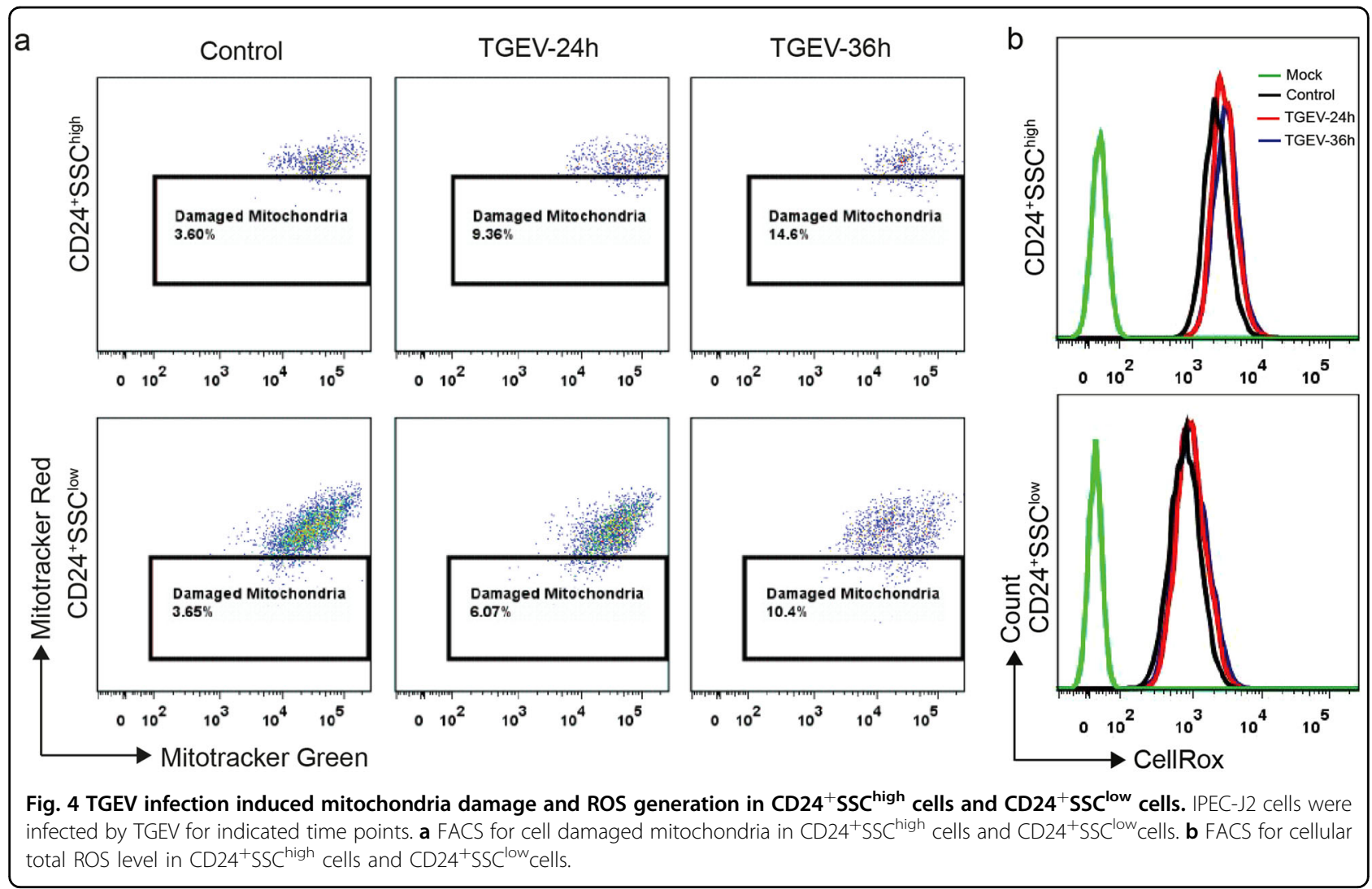

TGEV was mostly detected in $\mathrm{CD} 24^{+}$cells, which mainly include $\mathrm{CD}^{2} 4^{+} \mathrm{SSC}^{\text {high }}$ cells (Paneth cells) and $\mathrm{CD}_{24}{ }^{+} \mathrm{SSC}^{\text {low }}$ cells (enteroendocrine). In $\mathrm{CD}_{2} 4^{+} \mathrm{SSC}^{\text {low }}$ cells the effect was most pronounced, with the percentage of TGEV-positive cells nearly 10-fold as many as compared to other cell types in $24 \mathrm{~h}$ post TGEV-infected IPEC-J2 cells. However, a few cells were infected by TGEV in CD24- cells, which are mostly absorptive enterocytes (Fig. 5a). Although the difference of the infection proportion gradually narrowed in $36 \mathrm{~h}$ post infection, $\mathrm{CD} 24^{+} \mathrm{SSC}^{\text {low }}$ cells still contained more intracellular TGEV. Notably, less TGEV was detected in $36 \mathrm{~h}$ post infection Paneth cells, which is opposite with the result of $24 \mathrm{~h}$ post infection. These data suggest that TGEV selectively targets cell types for infection and replication. We followed those observations by examining TGEV infection rate over shorter time periods in several cell types. Surprisingly, in contrast to results from 24 and $36 \mathrm{~h}$ incubation, after only $1 \mathrm{~h}$ TGEV infection was higher in CD $24^{+} \mathrm{SSC}^{\text {high }}$ cells than $\mathrm{CD} 24^{+} \mathrm{SSC}^{\text {low }}$ or $\mathrm{CD} 24^{+} \mathrm{SSC}^{-}$. About $20 \%$ of $\mathrm{CD}_{2} 4^{+} \mathrm{SSC}^{\text {high }}$ cells carried TGEV, but only $2 \%$ cells were infected with TGEV in other cell types, such as all, CD24- and CD24 ${ }^{+}$SSC $^{\text {low }}$ IPEC-J2 cells (Fig. 5b). It suggests that TGEV selectively targets to $\mathrm{CD} 24^{+} \mathrm{SSC}^{\text {high }}$ cells for initial invasion.

\section{Aminopeptidase N (APN, CD13) receptor is required for TGEV invasion to Paneth Cells}

Similar to other coronaviruses (CoVs), TGEV utilizes APN (CD13) as its receptor for cell invasion (Supplementary Fig. S5b). Since TGEV mainly targets Paneth cells for initial invasion, we detected TGEV receptor APN expression in different cell types (Fig. 5c). FACS results showed only about $1.5 \%$ cells were CD13 positive in IPEC-J2 cells. However, $42.8 \% \quad \mathrm{CD} 24^{+} \mathrm{SSC}^{\text {high }}$ cells and $32.7 \%$ $\mathrm{CD} 24^{+} \mathrm{SSC}^{\text {low }}$ cells expressed CD13. Notably, almost no CD13 was expressed in other IPEC-J2 cells (CD24- cells) (Fig. 5c). Subsequently, removing $\mathrm{CD}^{+} 3^{+}$cells from IPEC-J2 cells by using FACS markedly decreased TGEV infection by $60 \%$. As expected, additional supplementation of $\mathrm{CD} 13^{+}$ cells in IPEC-J2 cells significantly promoted TGEV infection (Fig. 5d). Removing $\mathrm{CD} 13^{+}$cells didn't affect the number of $\mathrm{CD} 24^{+} \mathrm{SSC}^{\text {high }}$ and $\mathrm{CD} 24^{+} \mathrm{SSC}^{\text {low }}$ cells in $\mathrm{CD} 13^{+}$-removed IPEC-J2 cells, even upon TGEV infection (Supplementary Fig. S5a) and rescued Lgr5 ISCs loss, which was induced by TGEV (Supplementary Fig. S5b). In brief, TGEV initially invades Paneth cells through APN (CD13) receptor.

Subsequently, we found that inhibition of TGEV infection by $A P N$ gene knockout in IPEC-J2 cells rescues the fate of Lgr5 ISCs (Supplementary Fig. S6). This event directly inhibited TGEV infection and replication in 


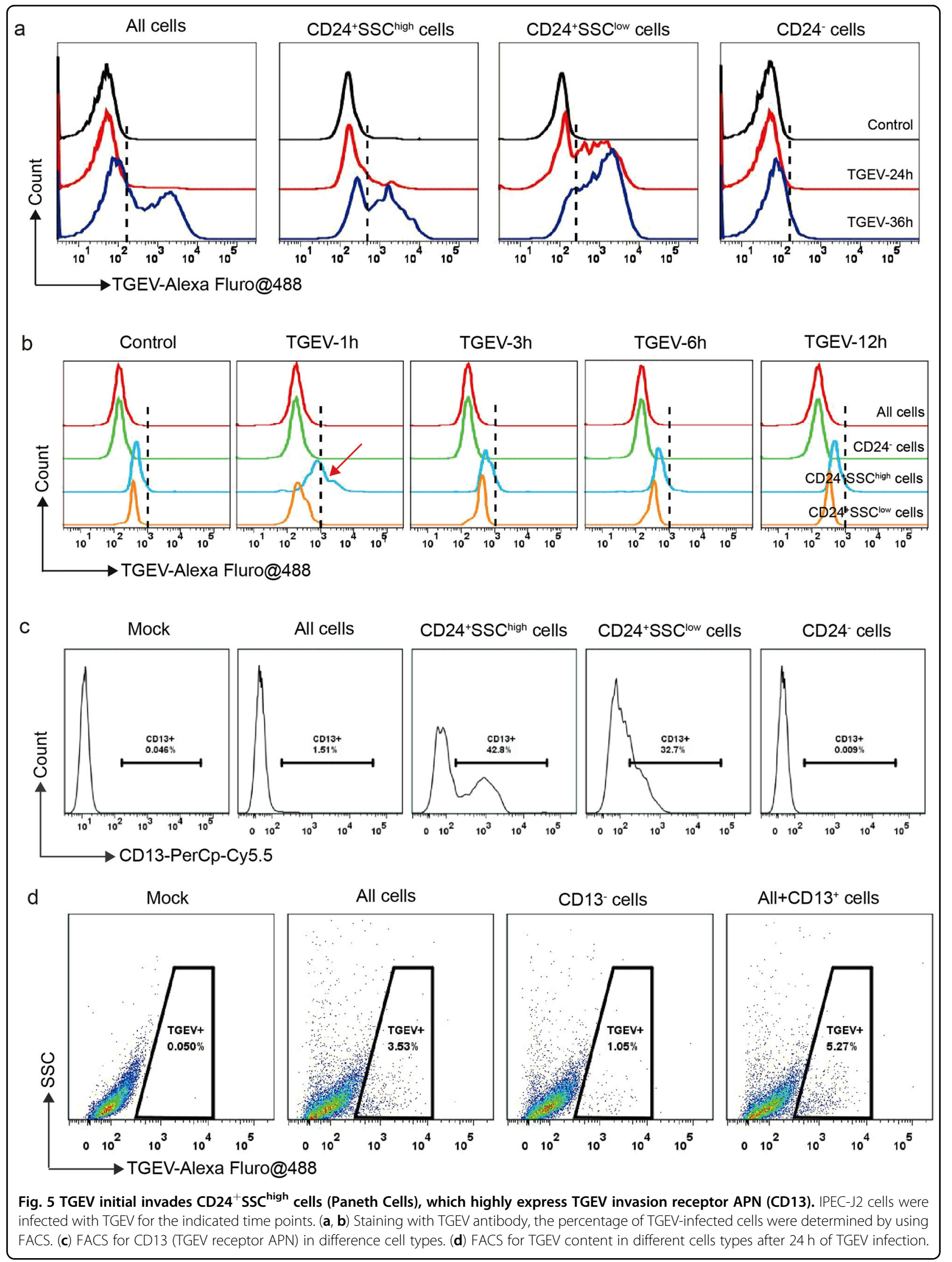




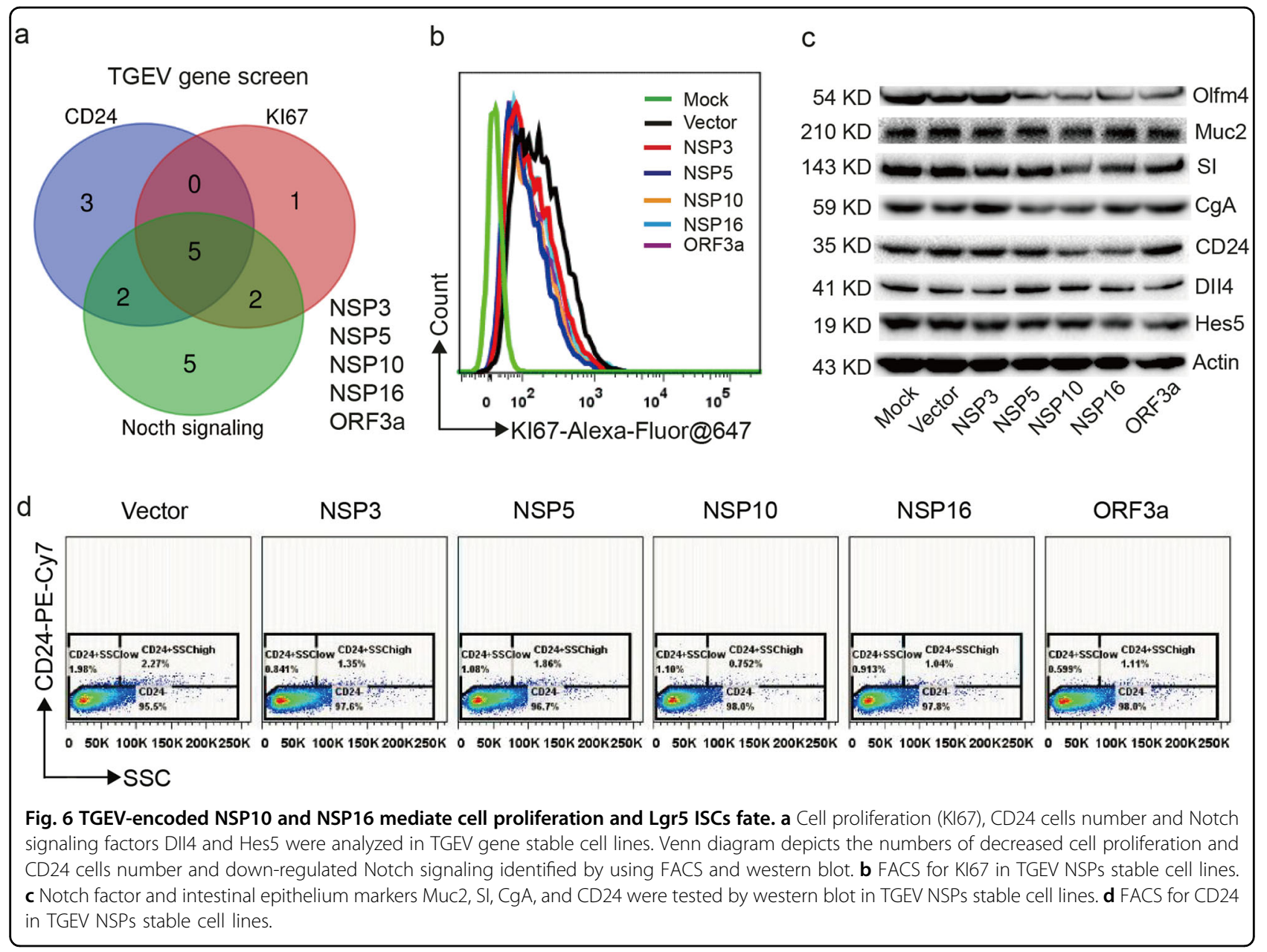

APN-KO IPEC-J2 cells (Supplementary Fig. S6b). Comparable to normal IPEC-J2 cells, APN-KO IPEC-J2 cells displayed inhibition on cell apoptosis, which was induced by TGEV in Lgr5 ISCs, CD24 ${ }^{+} \mathrm{SSC}^{\text {high }}, \mathrm{CD}^{2} 4^{+} \mathrm{SSC}^{\text {low }}$ and CD24- cells (Supplementary Fig. S6d; Supplementary Fig. S7b-d). Hence, there were no significant decreases in the number of Lgr5 ISCs, CD24 ${ }^{+} \mathrm{SSC}^{\text {high }}$ and $\mathrm{CD} 24^{+} \mathrm{SSC}^{\text {low }}$ cells, even with TGEV infection (Supplementary Fig. S6c and Fig. S7a).

TGEV non-structural protein 10 and 16 (NSP10 and NSP16) mediates cells proliferation and Lgr 5 ISCs fate decision

Naturally, viruses encoded proteins, which determine their epidemiological characteristic and pathogenesis, there is no exception for TGEV. To reveal which TGEVencoded protein mediates Lgr5 ISCs fate decision. 23 TGEV encode genes (NSP1-NSP16, ORF3a, ORF3b, $M$, $N, E, S$ and ORF7) were cloned and constructed within stable cell lines in IPEC-J2 cells. Cell proliferation (KI67 staining), CD24 cell number and Notch signaling (DII4 and Hes5 protein level) were tested in these stable cell lines. FACS for KI67 staining showed that there are 8
TGEV-encoded proteins that inhibit cell proliferation (Supplementary Fig. S8a), and 9 proteins that cause CD24 cells loss (Supplementary Fig. S8c). Moreover, there were 10 TGEV-encode proteins which down-regulated Notch signaling (Supplementary Fig. S8b). As represented via a Venn diagram, five TGEV encode proteins (NSP3, NSP5, NSP10, NSP16, and ORF3a) simultaneously affect cell proliferation, $\mathrm{CD} 24^{+}$cell number and Notch signaling (DII4 and Hes5 protein expression) (Fig. 6a). Among these viral proteins, NSP16 was the most significant inhibitor of cell proliferation and Notch signaling (Fig. 6b, c), and strongly decreased $\mathrm{CD} 24^{+} \mathrm{SSC}^{\text {high }}$ and CD24 ${ }^{+}$SSC $^{\text {low }}$ cell number (Fig. 6d). In addition, NSP16 down-regulated SI, CD24 and CgA protein level, but significantly up-regulated Muc2 protein expression (Fig. 6b). Moreover, NSP16 decreases Olfm4 expression in NSP16 stable cell lines (Fig. 6b). Similar results were observed in TGEV-infected jejunum (Fig. 2c-j). Therefore, we focused on NSP16, which mediated cell proliferation and Lgr5 ISCs fate decision. Of note, NSP10 induces similar phenomenon to NSP16 in IPEC-J2 cells (Fig. 6; Supplementary Fig. S8). 
TGEV-encoded NSP10/NSP16 protein complex not only interact with DII4 but also alter DII4 promoter activity

Normally, NSP16 activity is regulated by NSP10, and forms a protein complex with NSP10 to inhibit the translation and/or stability of host proteins in other CoVs, such as SARS-CoV and MERS-CoV. Moreover, TGEV and TGEV-encoded NSP10 and NSP16 distinctly inhibit Notch signaling factors (DII4 and Hes5). Therefore, we investigated whether TGEV-encoded NSP10 and/or NSP16 alter Notch signaling via interacting with these factors (DII4 and/or Hes5). To explore this point, we performed IP experiments in NSP10 and NSP16 stable transfected IPEC-J2 cell lines, and CO-IP experiments in NSP10 and NSP16 transiently transfected HEK239T cells. Anti-Flag immunoprecipitation followed by anti-DII4 and anti-HA western blotting showed specific binding of NSP10 and NSP16 to DII4 (Fig. 7a, b), but no interactions were detected between NSP10 and Hes5 as well as NSP16 and Hes5. To further test their interactions between NSP10, NSP16 and DII4, we utilized a Duolink proximity ligation assay (PLA), which can demonstrate proteinprotein interactions in situ by eliciting a fluorescent signal. PLA signals were detected in DII4-HA co-transfection with NSP10-Flag and/or NSP16-Flag cells, revealing the interaction of these proteins (Fig. 7c). We also tested for the interaction between TGEV-encoded NSP10 and NSP16 by using PLA. Indeed, NSP10 was observed to bind to NSP16 (Fig. 7c). To test whether the colocalization of NSP10, NSP16 and DII4 occurs, HEK239T cells were co-transfected with pLVX-DsRedMonomer-Flag-NSP10, pLVX-AcGFP1-Flag-NSP16, pLVX-mVenus-HA-DII4 and fixed at $36 \mathrm{~h}$ post transfection. The results showed that NSP16 not only co-localized with NSP10, but also co-localized with DII4 (Fig. 7d), which further confirmed the interaction between TGEVencoded NSP10, NSP16 and DII4. These results suggest that TGEV-encoded NSP10 and NSP16 form a complex in the host to alter DII4 protein level.

To reveal the potential mechanism of NSP10 and NSP16 in reducing DII4 protein level, we cloned a fragment spanning -310 bp to +1 bp (P1) of the DII4 predicted promoter into the pGL3-Basic vector (Fig. 7e). HEK293T cells were co-transfected with P1, Prl-TK (Renilla luciferase control reporter vectors), Vector, NSP10 and/or NSP16. We found that NSP10 robustly down-regulates DII4 promoter (P1) activity. However, NSP16 did not alter the transcriptional activity of DII4 promoter (Fig. 7f). Subsequently, we divided DII4 promoter (P1) into three sections (Fig. 7e) and detected the promoter activity of these fragments by using dualluciferase reporter system. NSP10 was observed to inhibit the promoter activity of three different DII4 promoter fragments by about $50-60 \%$ (Fig. 7g). Although NSP16 slightly enhanced the DII4 promoter (P2) activity by about $10 \%$, NSP10 still inhibited the DII4 promoter (P2) activity even in the presence of NSP16 (Fig. 7g).

\section{Discussion}

It is now well established that intestinal crypt cells respond to damage induced by high-dose irradiation or chemicals by activation of reserve stem cells ${ }^{5,25-27}$. Here, we reveal intestinal crypt cells exhibit a novel response to a diarrheal virus (Fig. 8). In this study we found that TGEV infection results in villous atrophy within $48 \mathrm{~h}$ and inhibits intestinal epithelium renewal by halting the selfrenewal and differentiation of Lgr5 ISCs. As the epithelium of the intestine is the fastest renewing tissue, sustained by Lgr $5 \mathrm{ISCs}^{28}$, once Lgr5 ISCs lose the ability of self-renewal and diferentiation, it will seriously affect intestinal epithelium turnover and perturb intestinal homeostasis. A recent report similarly showed that Heligmosomoides polygyrus infection causes Lgr5 ISCs loss through activating IFN- $\gamma$ generation and induces fetal-like reversion in the intestinal stem-cell niche ${ }^{4}$. Lgr5 ISCs are apoptosis sensitive cells to different types of stresses (such as ROS), so it is easy to be attacked ${ }^{29}$. Previous study demonstrated that TGEV-encoded N protein induced ROS generation, which contributes to cell apoptosis activation via p53 signaling in ST cells ${ }^{30}$. Although TGEV induces mildly ROS production in TGEV-infected IPEC-J2 cells, it robustly promotes ROS generation in Lgr5 ISCs (data not shown), which provides a potential explanation of Lgr5 ISCs loss and why more apoptosis cells were observed in crypt.

Except for cell apoptosis, TGEV-mediated Paneth cells loss also contributes to the developmental fate of Lgr5 ISCs. TGEV directly invades Paneth cells through the APN receptor, and then activates ROS generation, which ultimately induces Paneth cells apoptosis. Paneth cell loss severely affects the niche factors secretion (such as Wnt and Notch factors) needed for Lgr5 ISCs self-renewal and differentiation ${ }^{14}$. Among these niche factors, Notch factor is often linked to developmental patterning ${ }^{31}$, intestinal stem-cell self-renewal and crypt homeostasis ${ }^{20}$. Although a few studies shown that Paneth cells are dispensable for survival, proliferation, and stem-cell activity ${ }^{32,33}$. This because the other cell type severs the same function as paneth cells, such as mesenchymal cells ${ }^{14,34,35}$. Beyond stimulating Lgr5 ISCs cells with niche signals, Paneth cells also provide essential nutrients to $\mathrm{ISCs}^{1}$. Therefore, paneth cells are essential for Lgr5 ISCs. In our study TGEV infection significantly down-regulates Notch ligand DII4 and Notch effector Hes5 protein expression both in vitro and in vivo. Inactivation of DII4 causes the loss of stem and progenitor cells ${ }^{22}$. Thus, Paneth cells loss, affecting Notch signaling activation, may be the foundational mechanism of TGEV-mediated inhibition of Lgr5 ISCs self-renewal and differentiation. 


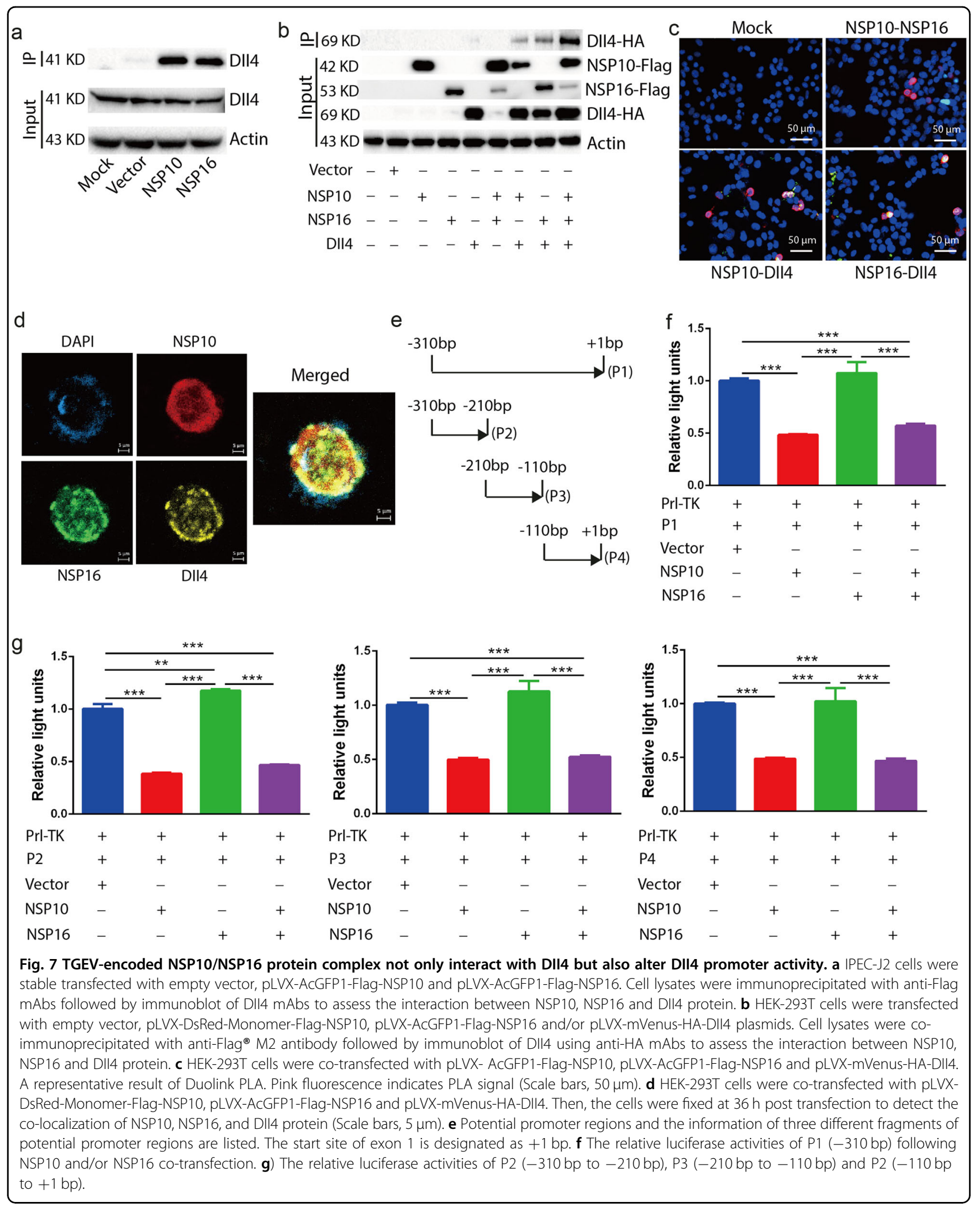




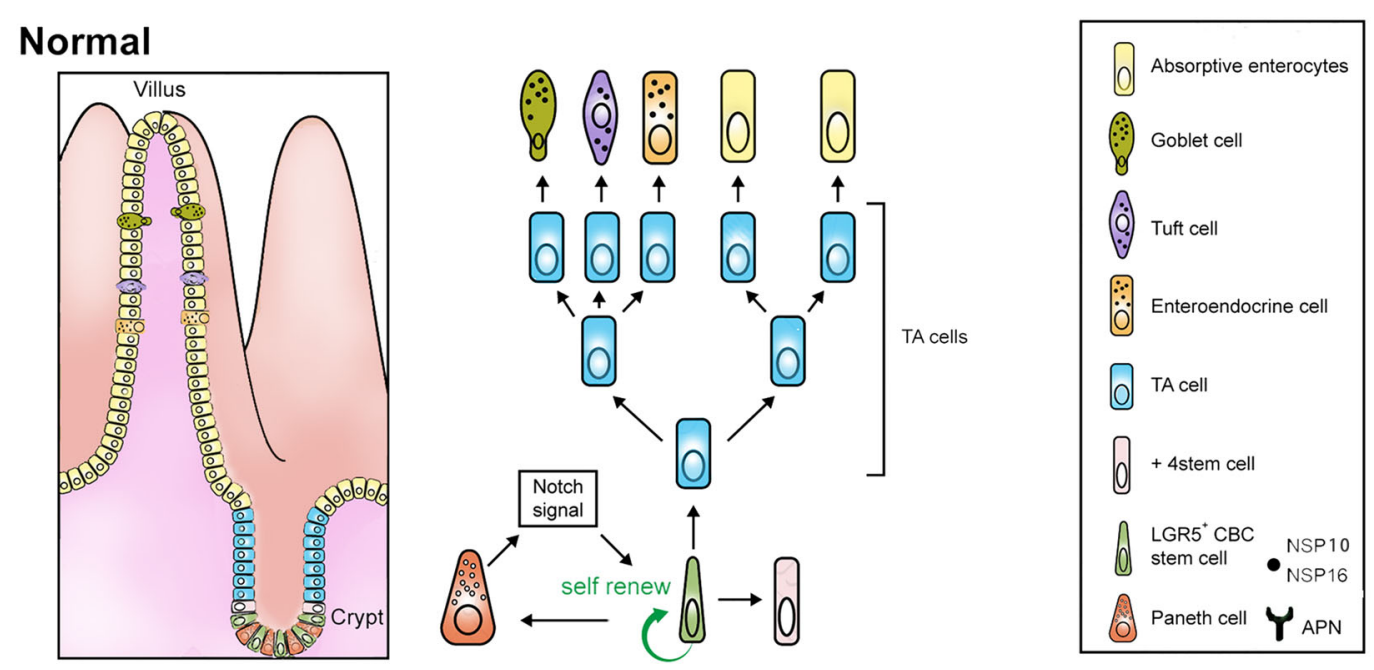

\section{TGEV Inflection}

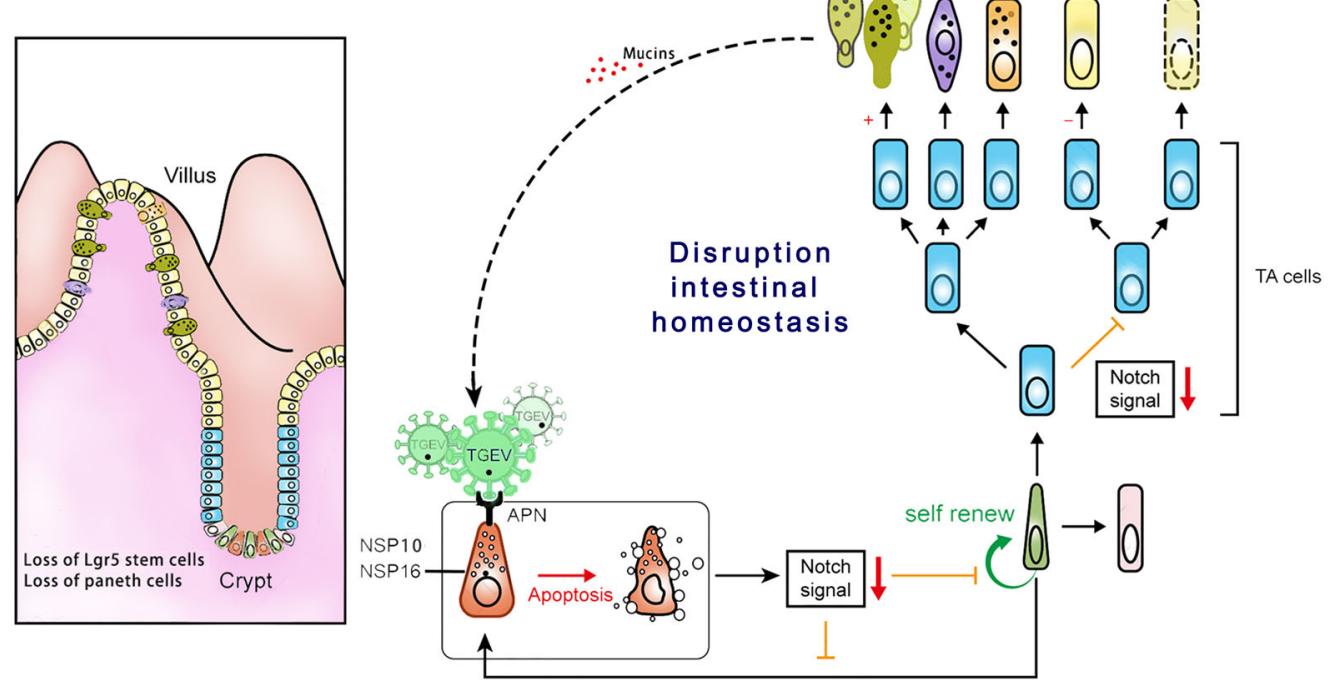

Fig. 8 Model of proposed mechanism of TGEV-mediated disruption of intestinal homeostasis. In the case of normal condition(the upper panel), Lgr5 ISCs continuously generate rapidly proliferating TA cells, which differentiate into the various functional cells on the villi (enterocytes, tuft cells, goblet cells and enteroendocrine cells) to replace the intestinal epithelial cells being lost via anoikis at the tip of villi. Paneth cells are unusual in that they intercalate with Lgr5 ISCs and provide niche factors (such as Notch signaling) for Lgr5 ISCs fate decision, which is essential for intestinal epithelial cells renewal and intestinal homeostasis maintenance. Upon TGEV infection (the lower panel), TGEV targets Paneth cells for initial invasion via APN receptor and robustly induces cell apoptosis in Paneth cells, which causes a dramatic loss of Paneth cells number and niche factor (Notch signaling) for Lgr5 ISCs self-renewal and differentiation. Notch signaling, a switch between secretory lineages and absorptive lineages differentiation, plays a vital function in intestinal cells fate decision. Notch signaling inhibition induced goblet cells differentiation at the cost of absorptive enterocytes. Interestingly, mucus secreted by goblet cells contains sialic acid, which promotes TGEV infection. Thus, as time goes on, the more TGEV invades intestinal epithelial cells, the more severe damage occurs in vivo. A vicious circle is gradually forms in TGEV-infected jejunum. This flawlessly explains why sharp villus atrophy occurs in TGEV-infected jejunum. The whole process is mediated by TGEV-encoded NSP10 and NSP16, which interactions regulate Lgr5 ISCs fate and intestinal homeostasis.

Notch signaling is a developmental switch for intestinal secretory cells and absorptive enterocytes ${ }^{2}$, as its suppression leads to a block of differentiation of enterocytes and a dramatic increase in the number of goblet cells ${ }^{23}$. As villus are mainly composed of enterocytes, TGEV infection most likely causes villous atrophy. Indeed, TGEV infection promotes progenitor cells differentiation to goblet cells at the cost of absorptive cells in TGEVinfected jejunum. TGEV infection causes villous atrophy within a short time (generally within $48 \mathrm{~h}$ ), and the villusheight/crypt-depth ratio rapidly changed from $7: 1$ to $1: 1^{36}$. Intriguingly, TGEV infection promotes goblet cells differentiation and accompanies an increase of mucins secretion. Contrary to the luminal protection of 
gastrointestinal tract, mucins have the ability to promote TGEV infection. As mucins are rich in sialic acids ${ }^{37}$, they are interaction partners for TGEV and thus may help to penetrate the mucus layer to gain access to APN on the surface of the intestinal epithelial cells ${ }^{38}$. Thus, over time, the more TGEV enters into intestinal epithelium, the more severe damage occurs in TGEV-infected jejunum. A circle promoting infection is then gradually formed within $24 \mathrm{~h}$ of TGEV infection. This is, at least in part, reason for villous sharp shortness and high mortality of TGEV infection. Thus, the mucins are a "double-edged sword" that needs to be balanced gently. Base on these results, it is not difficult to find that the disruption of TGEV mainly occur in crypt, and villous atrophy is the ultimate result of crypt damage. This "from bottom to top" finding of TGEV pathogenesis to intestine is quite novel to the traditional "top down" understanding of TGEV infection that villus damage occurs first and damage to crypt next. Together, our data suggests that the Notch pathway might represent the cell developmental "switch" of Lgr5 ISCs and Paneth cells in the case of TGEV infection. Therefore, Notch signaling may be an attractive therapeutic target for TGEV infection. The fact that Notch signaling controls key steps of differentiation in most and has become therapeutic targets for many diseases ${ }^{39}$.

Subsequently, we found that TGEV-encoded NSP16 significantly attenuates Notch signaling (DII4 and Hes5) activation. NSP16, a 2'-O-methyltransferase (2'-O-MTase) plays a crucial role for $2^{\prime}-O$-methylation of viral mRNA in capping of viral RNA, which permits viral infection with reduced host recognition ${ }^{40,41}$. The absence of 2'-O-methylation or NSP16 mutant activates a more robust type I IFN response that ablates viral infection and replication ${ }^{41,42}$. Thus, capping of viral RNA by NSP16 is an effective and successful strategy to disrupt host immune recognition. For TGEV, its encoded NSP16 not only helps itself to evade host recognition, which promotes TGEV infection and replication, but also inhibits Notch signaling via inhibiting the DII4 promoter activity, and ultimately disrupts intestinal homeostasis. Generally, COVs NSP16 activity is strictly dependent on its interaction with NSP10 $0^{42,43}$, this is no exception for TGEV. TGEV-encoded NSP10 interaction with NSP16 to prevent virus infection by cell innate immunity mechanisms. Inhibition of NSP10/NSP16 MTase activities by A MTase specific inhibitor AdoHcy significantly inhibits TGEV infection and replication (data not shown). Additionally, NSP10, NSP16 and their complex can interact with DII4, which normally binds to Notch receptors. This interaction may disturb Notch signaling. Notably, NSP10 not only binds to DII4, but also down-regulates DII4 promoter activity, which provides a good explanation of TGEV and its NSP10 and/or NSP16-mediated DII4 mRNA and protein decrease. Therefore, NSP10/NSP16 complex is likely another novel therapeutic target for TGEV infection.

In summary, our findings highlight a link between intestinal crypt cells response and virus infection that regulates Paneth cells function and Lgr5 ISCs fate. TGEV infection causes loss of Notch signaling, which inhibits Lgr5 ISCs self-renewal and differentiation and causes villous atrophy. This phenomenon can also be regarded as an explanation of TGEV pathogenicity, and possibly represents similar pathways used by other diarrheal virus (such as rotavirus) and bacteria, to disrupt intestinal homeostasis. Given its central role in TGEV infection, Notch signaling is an attractive therapeutic target for TGEV infection. Future studies will explore how intestinal crypt regenerates the damaged gut with diarrheal virus infection.

\section{Materials and methods \\ Animals and TGEV infection}

A total of 12 7-days-old DLY male piglets were obtained from a swine herd at Sichuan Agricultural University and artificially fed with milk for three days. After that, all piglets were randomly divided into two groups, the TGEV-infected group (TGEV) and the control group (Control) in this study $(n=6)$, and then piglets were orally inoculated with either 0 or $1 \times 10^{9}$ PFU TGEV (TCID50 $=10^{-7} / 100 \mu \mathrm{l}$ ) according to the previous assignment (Control vs. TGEV infected). Each experimental group of piglets was housed in a separate room in a high-security isolation facility. Piglets that developed significant diarrhea and lived two days after infection were used in the experiment. The experimental procedures used in this study were approved by the Animal Care Advisory Committee of Sichuan Agricultural University.

\section{Histological analysis, immunofluorescence and in fluorescence situ hybridization}

Intestinal tissue was collected and fixed overnight in $4 \%$ PFA in PBS and embedded in paraffin. In all, 5- $\mu \mathrm{m}$ sections were stained by HE and PAS for histological analysis and goblet cells staining. Sample were embedded in OCT and 8$\mu \mathrm{m}$ sections were performed for immunofluorescence staining by using the following primary antibodies: TUNEL (Roche, 11684817910), anti-Ki67 (BD; 550609), anti-CD24 (Thermo Fisher; MA5-11828), anti-Muc2 (Santa Cruz; sc515032), anti-CgA (Immunostar; 20086). All primary antibodies were used as 1:100 dilutions. Goat anti-mouse Alexa fluor ${ }^{\circledR} 488$ (Abcam, ab150113) and goat anti-mouse Alexa fluor ${ }^{\circledR} 647$ (ab150115) secondary antibodies were used at 1:500-1:1000 dilutions.

For fluorescence in situ hybridizations (FISH), tissues of jejunum were fixed overnight in 4\% PFA, paraffin embedded, and sectioned at $10 \mu \mathrm{m}$. The probe sequences 
targeting Lgr5, Olfm4 and Lys (Lysome) was: Lgr5 probe (5'-FAM-GACGACAGGCGGTTGGACGATAGGT-FA M-3'), Olfm4 probe (5'-FAM-CACTGACACCTCGCC ACCATTCCA-FAM-3') and Lys probe (5'-CY3-GCA CCGATCATAGACCTTGGCCTGTA-3'). The protocols used for in vitro transcription and in situ hybridization were previously described ${ }^{44}$. All images were acquired and processed with Zeiss Axio-Imager Z1 with apotome or Leica SP5 confocal microscope.

\section{Cell culture and viral infection}

IPEC-J2 cells were obtained from ATCC and cultured in DMEM/F12 supplemented with $10 \% \mathrm{FBS}, 100 \mathrm{IU} / \mathrm{ml}$ of penicillin, $100 \mathrm{mg} / \mathrm{ml}$ of streptomycin, $5 \mathrm{mg} / \mathrm{ml} \mathrm{hEGF}$ and $10 \mathrm{nM}$ HEPES, at $37^{\circ} \mathrm{C}$ in a $5 \% \mathrm{CO} 2$ atmosphere incubator. TGEV was provided by Prof. Zhiwen Xu. Confluent (70\%) IPEC-J2 cells were inoculated with TGEV at MOI of $1 \mathrm{for} 1 \mathrm{~h}$ at $37^{\circ} \mathrm{C}$. The unattached virus were removed and the cells were washed one time with PBS. Subsequently, fresh growth medium was added.

\section{TGEV gene screen and stable cell lines constructs}

The TGEV genes were constructed by RT-PCR amplification from the genomic RNA of TGEV strain WH-1 and cloned into lentiviral vector pLVX-AcGFP1-N1. All plasmids were confirmed by sequencing and transfected into HEK293T packaging cells with pLP/VSVG and psPAX2 two plasmids packing system. Stably transfected IPEC-J2 cells were selected with $2.5 \mu \mathrm{g} / \mathrm{ml}$ puromycin selection for 2 weeks. Selected cells were tested for TGEV genes mRNA expression by reverse transcription-PCR (RT-PCR).

\section{Flow cytometry and cell sorting}

Flow cytometry (FACS) experiments were performed with the following antibody: Alexa Fluor ${ }^{\circledR} 647$-Lgr5 (BD; 562912), FITC-CD24 (BD; 555427), PE-Cy7TM-CD24 (BD; 561646), PerCP-CyTM 5.5-CD13 (BD; 561361). FACS measurements were performed using a BD-FACS service device and analyzed with FlowJo software (FlowJo LLC). MFI is defined as the difference in the signal intensity between an unstained control and a stained sample. For sorting, a FACS Aria SORP device (Becton Dickinson) was used.

\section{Quantification of reactive oxygen species and damaged mitochondria}

For the detection of ROS, $5 \mu \mathrm{M}$ CellRox Deep Red reagent (Life Technologies; C10422) was incubated with IPEC-J2 cells culture for $30 \mathrm{~min}$ at $37^{\circ} \mathrm{C}$. Dysfunctional mitochondria were monitored by fluorescence levels upon staining with $100 \mathrm{nM}$ MitoTracker Green FM (Life Technologies; M7514) and $100 \mathrm{nM}$ MitoTracker Red CMXRos (Life Technologies; M7512) for $30 \mathrm{~min}$ at $37^{\circ} \mathrm{C}$.
Subsequently, cells were washed three times with PBS and analyzed by flow cytometry.

\section{Cell apoptosis and proliferation}

Apoptotic cell death was detected by FITC-, Alexa Fluor ${ }^{\circledR} 647$ - conjugated Annexin V with propidium iodide (PI) staining assay (Biolegend; 640914 and 610912) after the manufacturer's protocols. Briefly, IPEC-J2 cells were harvested, then $10^{6}$ cells were resuspended in $100 \mu \mathrm{L} 1 \times$ binding buffer followed by incubation with $2 \mu \mathrm{L} / 10^{6}$ cells Annex V per test for 20 min on ice. Subsequently, $400 \mu \mathrm{L}$ $1 \times$ binding buffer and $1 \mu \mathrm{L} \mathrm{PI}(1 \mathrm{mg} / \mathrm{ml})$ was added to the sample and immediately analysis by FACS. Cell proliferation was determined by using FACS. IPEC-J2 cells infected with TGEV were harvested and fixed with $4 \%$ formalin in PBS for $15 \mathrm{~min}$ at room temperature. Fixed cells were permeabilized with $0.05 \%$ Triton X-100 in PBS at room temperature for $15 \mathrm{~min}$. For intracellular Ki67 (BD; 550609) staining, cells were incubated for $2 \mathrm{~h}$ with Ki67 antibody $\left(0.5 \mu \mathrm{g} / 10^{6}\right.$ cells), followed by secondary antibody staining with 1:1000 dilution of goat anti-mouse Alexa fluor ${ }^{\circledR} 488$ (Abcam; ab150113) for additional $1 \mathrm{~h}$.

\section{qPCR and western blotting}

Total RNA was extracted from samples using TRIzol reagent (Invitrogen) according to the manufacturer's protocol and cDNA synthesised using the prime script ${ }^{\mathrm{TM}}$ RT reagent kit with gDNA eraser (Takara, RR047A). Relative gene expression was calculated with the $\Delta \Delta C_{T}$ method, normalizing the results to the value for the Gapdh gene. Protein extraction and western blotting were performed as described previously, using primary antibodies against Olfm4 (Abcam; ab85046), SI (Santa Cruz; sc-27603), CD24 (Thermofisher; MA5-11828), Muc2 (Santa Cruz; sc-515032), CgA (ImmunoStar; 20086), Hes5 (Santa Cruz; sc-293445), DII4 (Abcam; ab7280) and $\beta$-Actin (Santa Cruz; 47778). The dilution of primary antibodies was 1:1000. The second goat anti-rabbit and goat anti-mouse antibodyies conjugated to HPR (Santa Cruz; sc-2030 and sc-2031) was used (1:3000).

\section{CRISPR-Cas9 knockout of pANPEP}

The porcine ANP gene (gene ID: 397520) was disrupted in IPEC-J2 cells by using the CRISPR-Cas9 technique. In brief, two guide RNAs (gRNAs) targeting the first exon of the ANP gene, GGTAGGCGGTACCGGTTCCA (ANPKO-1) and GCGTTGTGGGTAGGCGGTAC (ANP-KO2 ), and complementary oligonucleotides were designed. The annealed gRNA duplexes were cloned into the lentiCRISPRv2 vector (Addgene; 52961) by using the BsmBI restriction site. The gRNA-expressing vectors were transfected into HEK239T cells, and cell culture supernatants containing viral particles were harvested and used for infection of IPEC-J2 cells. IPEC-J2 cells were 
subsequently subjected to puromycin selection for 2 weeks. Selected clones were tested for ANP mRNA expression by reverse transcription-PCR (RT-PCR). A representative clone from each of the two CRISPR constructs was used in the experiments described here.

\section{Immunoprecipitation (IP) and co-immunoprcipitation (CO-}

IP)

NSP10 and NSP16 stable IPEC-J2 cells were harvested and lysed in lysis buffer. $200 \mu \mathrm{g}$ of cell lysate protein was incubated with $2.5 \mu \mathrm{g}$ anti-Flag ${ }^{\circledR} \mathrm{M} 2$ antibody (CST; 14793) and $20 \mu$ of protein

A magnetic beads (CST; 73778) overnight at $4{ }^{\circ} \mathrm{C}$. Samples were washed three times with lysis buffer, resuspended in $2 \mathrm{x}$ sample buffer and analyzed with immunoblot analysis using DII4 antibody. For CO-IP experiments, Hek293T cells expressing NSP10-Flag, NSP16-Flag and/or DII4-HA constructs were harvested and lysed in lysis buffer. Samples were then centrifuged to remove precipitated proteins and were incubated with anti-Flag ${ }^{\circledR}$ M2 antibody overnight at $4{ }^{\circ} \mathrm{C}$. Samples were washed three times with lysis buffer, resuspended in $2 \mathrm{x}$ sample buffer and analyzed with immunoblot analysis using the indicated antibodies.

\section{Luciferase reporter assay}

HEK293T cells were seed into 48 -well plates at $3 \times 10^{5}$ cells/well and co-transfected with different DII4 predicted promoter plasmids, NSP10 and NSP16. All cells were transfected with the Renilla luciferase control plasmid PrlTK (Promega, E2241). Luciferase activity was measured with a Dual-Luciferase assay kit (Beyotime; RG027) with Glomax 96 microplate luminometer (Promega) in luminometer mode following the manufacturer's proto$\mathrm{col}$. The raw values of firefly luciferase were normalized to Renilla luciferase.

\section{Duolink proximity ligation assay}

HEK239T cells expressing NSP10-HA, NSP16-Flag or DII4-HA construct were co-cultured on a cover slip. Cells were fixed with $4 \%$ PFA for 15 min at room temperature, blocked with 0.1 gelation-PBS, and stained with 1:250 rabbit anti-Flag and 1:250 mouse anti-HA antibodies for $1 \mathrm{~h}$ at room temperature. Cells were then stained with 1:000 donkey anti-rabbit plus and donkey anti-mouse minus second antibodies. After washing with $0.1 \%$ gelatinPBS, proximity ligation assay (PLA) was done according to manufacturer's protocol (Sigma).

\section{Statistical analysis}

Each of the experiments described here was performed in at least three independent biological replicates. Statistical analysis was performed by using Graph Pad Prism software. All results were unpaired two-tailed Student's $T$ test and/or one-way analysis of variance (ANOVA). $P \leq$ 0.05 were considered to be statistically significant $(" p<$ $0.05,{ }^{* * *} p<0.01,{ }^{* * * * *} p<0.001$ ).

\section{Acknowledgements}

This work was supported by China National Key Research \& Development Project (2016YFD0501204) and China Agricultural Research System on Pigs (CARS-35).

\section{Author details}

${ }^{1}$ Institute of Animal Nutrition, Sichuan Agricultural University, Chengdu, China ${ }^{2}$ Key Laboratory for Animal Disease-resistance Nutrition of China Ministry of Education, Sichuan Agricultural University, Chengdu, China. ${ }^{3}$ College of Veterinary Medicine, Sichuan Agricultural University, Chengdu, China

\section{Author contributions}

A.W., L.W., J.Z., J.L.,Y.L., J.Y., X.M., P.Z., L.C. and Z.H: performed experiments. A.W., B.Y., Z.W. and D.C.: designed experiments. A.W., B.Y., J.H., D.W. and D.C.: wrote the paper. A.W., B.Y., K.Z. and D.C.: interpreted data and modified the paper.

\section{Conflict of interest}

The authors declare that they have no conflict of interest.

\section{Publisher's note}

Springer Nature remains neutral with regard to jurisdictional claims in published maps and institutional affiliations.

Supplementary Information accompanies this paper at (https://doi.org/ 10.1038/s41419-020-2233-6).

Received: 13 September 2019 Revised: 2 January 2020 Accepted: 2 January 2020

Published online: 20 January 2020

\section{References}

1. Rodriguez-Colman, M. J. et al. Interplay between metabolic identities in the intestinal crypt supports stem cell function. Nature 543, 424-427 (2017).

2. Clevers, H. The intestinal crypt, a prototype stem cell compartment. Cell 154, 274-284 (2013).

3. Rathinam, V. A. K. \& Chan, F. K. Inflammasome, inflammation, and tissue homeostasis. Trends Mol. Med. 24, 304-318 (2018).

4. Nusse, Y. M. et al. Parasitic helminths induce fetal-like reversion in the intestinal stem cell niche. Nature 559, 109-113 (2018).

5. van Es, J. H. et al. Dll1+ secretory progenitor cells revert to stem cells upon crypt damage. Nat. Cell Biol. 14, 1099-1104 (2012).

6. Yan, K. S. et al. Intestinal enteroendocrine lineage cells possess homeostatic and injury-inducible stem cell activity. Cell Stem Cell 21, 78-90 e76 (2017).

7. Zhao, $X$. et al. microRNA-4331 promotes transmissible gastroenteritis virus (TGEV)-induced mitochondrial damage via targeting RB1, upregulating interleukin-1 receptor accessory protein (IL1RAP), and activating p38 MAPK pathway in vitro. Mol. Cell. Proteom. 17, 190-204 (2018).

8. Almazan, F., Galan, C. \& Enjuanes, L. The nucleoprotein is required for efficient coronavirus genome replication. J. Virol. 78, 12683-12688 (2004).

9. Xia, L., Yang, Y., Wang, J., Jing, Y. \& Yang, Q. Impact of TGEV infection on the pig small intestine. Virol. J. 15, 102 (2018).

10. Huang, C. et al. Alphacoronavirus transmissible gastroenteritis virus nsp1 protein suppresses protein translation in mammalian cells and in cell-free HeLa cell extracts but not in rabbit reticulocyte lysate. J. Virol. 85, 638-643 (2011).

11. Charley, B. \& Laude, H. Induction of alpha interferon by transmissible gastroenteritis coronavirus: role of transmembrane glycoprotein E1. J. Virol. 62, 8-11 (1988).

12. Zhou, Y. et al. Cellular RNA helicase DDX1 is involved in transmissible gastroenteritis virus nsp14-induced interferon-beta production. Front. Immunol. 8, 940 (2017).

13. Xue, M. et al. IL-22 suppresses the infection of porcine enteric coronaviruses and rotavirus by activating STAT3 signal pathway. Antivir. Res. 142, 68-75 (2017). 
14. Barker, N. Adult intestinal stem cells: critical drivers of epithelial homeostasis and regeneration. Nat. Rev. Mol. Cell Biol. 15, 19-33 (2014).

15. Barker, N. et al. Identification of stem cells in small intestine and colon by marker gene Lgr5. Nature 449, 1003-1007 (2007).

16. Date, S. \& Sato, T. Mini-gut organoids: reconstitution of the stem cell niche. Annu. Rev. Cell Dev. Biol. 31, 269-289 (2015).

17. van der Flier, L. G. et al. Transcription factor achaete scute-like 2 controls intestinal stem cell fate. Cell 136, 903-912 (2009).

18. Dayton, T. L. \& Clevers, H. Beyond growth signaling: paneth cells metabolically support ISCs. Cell Res. 27, 851-852 (2017).

19. Sato, T. et al. Paneth cells constitute the niche for Lgr 5 stem cells in intestinal crypts. Nature 469, 415-418 (2011).

20. VanDussen, K. L. et al. Notch signaling modulates proliferation and differentiation of intestinal crypt base columnar stem cells. Development 139 488-497 (2012).

21. Ueo, T. et al. The role of Hes genes in intestinal development, homeostasis and tumor formation. Development 139, 1071-1082 (2012).

22. Pellegrinet, L. et al. Dll1- and dll4-mediated notch signaling are required for homeostasis of intestinal stem cells. Gastroenterology 140, 1230-1240 (2011). e1231-1237.

23. van Es, J. H. et al. Notch/gamma-secretase inhibition turns proliferative cells in intestinal crypts and adenomas into goblet cells. Nature 435, 959-963 (2005).

24. Milano, J. et al. Modulation of notch processing by gamma-secretase inhibitors causes intestinal goblet cell metaplasia and induction of genes known to specify gut secretory lineage differentiation. Toxicological Sci. 82, 341-358 (2004).

25. Yin, X. et al. Niche-independent high-purity cultures of Lgr5+ intestinal stem cells and their progeny. Nat. Methods 11, 106-112 (2014).

26. Tetteh, P. W. et al. Replacement of lost Lgr5-positive stem cells through plasticity of their enterocyte-lineage daughters. Cell Stem Cell 18, 203-213 (2016).

27. Li, B. et al. Impaired Wnt/beta-catenin pathway leads to dysfunction of intestinal regeneration during necrotizing enterocolitis. Cell Death Dis. 10, 743 (2019).

28. Schuijers, J., van der Flier, L. G., van Es, J. \& Clevers, H. Robust cre-mediated recombination in small intestinal stem cells utilizing the olfm4 locus. Stem cell Rep. 3, 234-241 (2014).

29. Bigarella, C. L., Liang, R. \& Ghaffari, S. Stem cells and the impact of ROS signaling. Development 141, 4206-4218 (2014).
30. Ding, L. et al. TGEV nucleocapsid protein induces cell cycle arrest and apoptosis through activation of p53 signaling. Biochem. Biophys. Res. Commun. 445 497-503 (2014).

31. Kopan, R. \& llagan, M. X. The canonical Notch signaling pathway: unfolding the activation mechanism. Cell 137, 216-233 (2009).

32. Kim, T. H., Escudero, S. \& Shivdasani, R. A. Intact function of Lgr5 receptorexpressing intestinal stem cells in the absence of Paneth cells. Proc. Natl Acad. Sci. USA 109, 3932-3937 (2012).

33. Farin, H. F., Van Es, J. H. \& Clevers, H. Redundant sources of Wnt regulate intestinal stem cells and promote formation of Paneth cells. Gastroenterology 143, 1518-1529 e1517 (2012).

34. Gehart, H. \& Clevers, H. Tales from the crypt: new insights into intestinal stem cells. Nat. Rev. Gastroenterol. Hepatol. 16, 19-34 (2019).

35. Valenta, T. et al. Wnt ligands secreted by subepithelial mesenchymal cells are essential for the survival of intestinal stem cells and gut homeostasis. Cell Rep. 15, 911-918 (2016).

36. Pensaert, M., Haelterman, E. O. \& Burnstein, T. Transmissible gastroenteritis of swine: virus-intestinal cell interactions. I. Immunofluorescence, histopathology and virus production in the small intestine through the course of infection. Arch. Gesamt. Virusforsch. 31, 321-334 (1970).

37. Johansson, M. E., Sjovall, H. \& Hansson, G. C. The gastrointestinal mucus system in health and disease. Nat. Rev. Gastroenterol. Hepatol. 10, 352-361 (2013).

38. Schwegmann-Wessels, $\mathrm{C}$. et al. The sialic acid binding activity of the $\mathrm{S}$ protein facilitates infection by porcine transmissible gastroenteritis coronavirus. Virol. J. 8, 435 (2011).

39. Siebel, C. \& Lendahl, U. Notch signaling in development, tissue homeostasis, and disease. Physiological Rev. 97, 1235-1294 (2017).

40. Menachery, V. D., Debbink, K. \& Baric, R. S. Coronavirus non-structural protein 16: evasion, attenuation, and possible treatments. Virus Res. 194, 191-199 (2014).

41. Daffis, $\mathrm{S}$. et al. 2'-O methylation of the viral mRNA cap evades host restriction by IFIT family members. Nature 468, 452-456 (2010).

42. Menachery, V. D. et al. Middle east respiratory syndrome coronavirus nonstructural protein 16 is necessary for interferon resistance and viral pathogenesis. mSphere 2, https://doi.org/10.1128/mSphere.00346-17 (2017).

43. Decroly, E. et al. Coronavirus nonstructural protein 16 is a cap-0 binding enzyme possessing (nucleoside-2'O)-methyltransferase activity. J. Virol. 82 8071-8084 (2008).

44. Gregorieff, A. et al. Expression pattern of Wnt signaling components in the adult intestine. Gastroenterology 129, 626-638 (2005) 